\title{
Factores asociados a la calidad de vida en el final de la vida
}

\author{
Factors associated with quality of life at the end of life
}

\section{Objetivo}

Determinar los factores que ejercen mayor influencia sobre la calidad de vida al final de la vida (CVFV) y desarrollar un modelo para predecirla.

\section{Diseño y Lugar}

Cohorte prospectiva y multicéntrica. Tomó lugar en centros hospitalarios de New Haven, West Haven, Dallas, Boston y Hooksett (EE.UU.) entre 2002 y 2008.

\section{Pacientes}

396 pacientes con cáncer resistente a la quimioterapia, metástasis y esperanza de vida menor a seis meses, mayores de 20 años de edad y que contaban con un cuidador informal (familiar). Fueron excluidos los cuidadores o pacientes con deterioro cognitivo. La edad promedio fue 58,7 años $( \pm 12,5)$, (65\% eran caucásicos y $71,3 \%$, cristianos). El $60,8 \%$ tenía cobertura en salud y el $52,4 \%$, educación secundaria. Fueron seguidos hasta la muerte, ocurrida en promedio a los 4,1 meses.

\section{Evaluación de factores pronósticos y medición de resulta- dos principales}

En sendas entrevistas iniciales realizadas al paciente y a sus cuidadores fueron evaluadas varios aspectos: autosuficiencia, estilos de afrontamiento, religiosidad/espiritualidad, existencia de directivas anticipadas e indicaciones de no resucitar, visitas pastorales durante la internación y uso de servicios de salud mental. Fueron utilizadas entrevistas estructuradas para depresión, trastorno de ansiedad, estrés postraumático y trastorno de pánico, así como evaluaciones validadas sobre la relación médico-paciente. El estado clínico y las comorbilidades fueron medidos a través de la escala de Karnofsky y del índice de Charlson, utilizándose el cuestionario McGill y subescalas de carga de síntomas y de apoyo social, así como un componente de la escala del Instituto Nacional del Envejecimiento/Medición Multidimensional de Fetzer para religiosidad/espiritualidad.
Zhang B y col. Arch Intern Med. 2012;172(15):1133-1142.

Entre las dos y las tres semanas posteriores a la muerte fueron nuevamente entrevistados los cuidadores para indagar sobre su estado y sobre la asistencia recibida durante la última semana de vida.

\section{Resultados principales}

Si bien la mayor parte de la variabilidad de la CVFV no pudo ser explicada por los factores evaluados, fueron identificaron algunos débiles predictores:

1) la permanencia en una unidad de cuidados intensivos explicó en sentido negativo el $4,4 \%$ de la variabilidad de la CVFV y la muerte en el ámbito hospitalario, el 2,7\%; 2) la preocupación del paciente reportada al inicio el 2,7\% y la no práctica de oraciones religiosas o meditación al inicio, el 2,5\%, 3) el lugar donde recibió el tratamiento del cáncer el 1,8\%, 4) el uso de sonda de alimentación el 1,1\%,7) la no recepción de cuidado pastoral durante la internación institucional el 1,0\%, 5) el uso de quimioterapia el $0,8 \%$, y 6 ) el no sentir una alianza terapéutica médico-paciente, al inicio $(0,7 \%)$.

Los pacientes con menor sobrevida y los más jóvenes tuvieron peor CVFV, y un mejor estado de salud de los cuidadores se asoció con una mejor CVFV de los pacientes. Los cuidadores informales (familiares) calificaron la CVFV de los pacientes significativamente peor que los cuidadores formales (profesionales).

\section{Conclusiones}

Si bien la asociación es débil, los pacientes con cáncer avanzado que logran evitar las hospitalizaciones y las estancias en unidades de cuidados intensivos, que logran estar menos preocupados, que rezan o meditan, que reciben la visita de un pastor durante la internación, y que sienten una alianza terapéutica con sus médicos, tienen mejor CVFV que los que no lo logran.

Fuentes de financiamiento: Instituto Nacional de Salud Mental, Instituto Nacional del cáncer y Centro de Epidemiología Psicosocial e Investigación de Resultados del Instituto del Cáncer Dana Farber (EEUU).

\section{Comentario}

Si bien el final de la vida empieza a ser un tema de interés para la investigación en servicios de salud, las cuestiones vinculadas a la CVFV constituyen un grupo significativamente menor de las investigaciones publicadas. Por ejemplo, luego de realizar una sencilla búsqueda en PubMed encontramos 650 artículos publicados durante los últimos cinco años que hayan incluido en su título los términos "final de la vida", y sólo 18 que además contengan la expresión "calidad de vida".

Sin duda existen dificultades metodológicas importantes, pero parece obvio que para la mayoría de los médicos, para los servicios de salud, y para gran parte de los usuarios, el de la muerte es un tema de difícil abordaje, probablemente por complejas cuestiones de orden psicológico (en lo individual), de orden cultural (en lo colectivo), y de orden económico (para los sistemas de salud).

\section{Conclusiones del comentador}

El trabajo que hoy comentamos implica un esfuerzo por comprender sistemáticamente qué podemos ofrecer a las personas que están muriendo. Como ya sabemos, no es posible extrapolar sus conclusiones, que resultan muy plausibles, particularmente en lo que respecta a las diferencias respecto de la religiosidad o espiritualidad entre la población del estudio y las nuestras.

Pero, al tiempo que nos plantea este interrogante, también reafirma la necesidad de repensar las intervenciones del equipo de salud ante estos pacientes a los que acompañamos a morir, y enfocarnos en evitar "medicalizar" la última etapa de la vida, que es la muerte'.

Javier Vilosio [ Servicio de Clínica Médica y Departamento de Salud Pública del Instituto Universitario Hospital Italiano de Buenos Aires, javier.vilosio@ hospitalitaliano.org.ar ]

Vilosio J. Factores asociados a la calidad de vida en el final de la vida. Evid Act Pract Amb. 16(1) Ene-Mar 2013. 11. Comentado de: Zhang B y col. Factors Important to Patients' Quality of Life at the End of Life. Arch Intern Med. 2012 Aug 13; 172(15):1133-42. PMID: 22777380.

\section{Referencias}

1. Mainetti JA. La medicalización de la vida. Electroneurobiología 2006; 14 (3):71-89. Disponible en: http://electroneubio.secyt.gov.ar/medicalizacion_de_la_vida.htm 